\title{
Microglial activation after ischaemic stroke
}

To cite: Zhang S. Microglial activation after ischaemic stroke. Stroke and Vascular Neurology 2019;4: e000196. doi:10.1136/svn-2018-000196

Received 19 0ctober 2018 Revised 16 April 2019 Accepted 23 April 2019 Published Online First 10 May 2019

Check for updates

(c) Author(s) (or their employer(s)) 2019. Re-use permitted under CC BY-NC. No commercial re-use. See rights and permissions. Published by BMJ.

Gansu Key Laboratory of Biomonitoring and Bioremediation for Environmental Pollution, School of Life Sciences, Lanzhou University, Lanzhou, China

Correspondence to Dr Shengxiang Zhang, Gansu Key Laboratory of Biomonitoring and Bioremediation for Environmental Pollution, Lanzhou University School of Life Sciences, Lanzhou, China; sxzhang@|zu.edu.cn

\section{ABSTRACT}

Ischaemic stroke can induce rapid activation of microglia. As the resident immune cells of the central nervous system, microglial activation is believed to play a central role in neuroinflammation and pathological progression of ischaemic tissue. The activation of microglia after ischaemia involves several stereotypical events including morphological transformation, proliferation and polarisation. Studies using confocal or two-photon imaging techniques have revealed that the degree of microglial activation is correlated with the degree of ischaemia. Activated microglia display diverse polarisation phenotypes. It remains largely unclear regarding whether activated microglia are beneficial or detrimental to poststroke recovery. This mini-review focuses on the morphological and functional aspects of microglial activation, with particular attention to progress in twophoton imaging studies.

\section{INTRODUCTION}

Microglia are the resident immune cells in the central nervous system (CNS). Ontogenically, they are myeloid lineage cells derived from the yolk sac and colonise the CNS during early development. ${ }^{12}$ Unlike cortical neurons, microglia have the potential to proliferate and can be replenished through self-renewal. ${ }^{3} 4$ Microglia in physiological conditions display ramified morphology. The fine processes of microglia are highly dynamic and actively monitor the microenvironment. ${ }^{56}$ It is generally believed that microglia serve a critical role in immune surveillance and maintaining CNS homeostasis. In addition, microglia are also involved in circuit rewiring and synapse plasticity in the brain. ${ }^{78}$

Abundant data suggest that microglia are not only important for normal brain function but also crucial for neuroinflammation and pathogenesis in neurological diseases. ${ }^{9} 10$ Microglia switch from homeostatic to the activated state in response to pathological stimuli. The activation of microglia involves several phenotypic changes in morphology and function including morphological transformation, proliferation and polarisation. The degree of activation varies depending on the nature and severity of the pathological conditions and is well described in many pathological conditions such as traumatic brain injury, prion disease and Alzheimer's disease. ${ }^{11-13}$ Progressive neurodegeneration is often associated with chronic microglial activation which can take weeks or even years, ${ }^{14}$ but acute injury can induce rapid extension of microglial processes within minutes. ${ }^{5}$ In the case of ischaemic stroke, reduction in blood flow can initiate rapid deramification of microglia, and severe ischaemia is accompanied by an intense reaction of microgliosis. ${ }^{1516}$ Microglial activation is believed to be involved in the pathological progression of ischaemic tissue. However, the function of activated microglia in ischaemic stroke remains not completely understood. Previous studies indicate that microglia can be neuroprotective or neurotoxic to ischaemic brain. ${ }^{17} 18$ The different results in the literature suggest that distinct events may occur during the complicated process of microglial activation in different ischaemic conditions. Much of our understanding of microglial activation is obtained using high-resolution imaging techniques including confocal and two-photon microscopy. Confocal microscopy enables the imaging of live cells with submicron resolution and is well suited for monitoring microglial activation in vitro. However, due to several factors such as light scattering and phototoxicity, confocal microscopy is not ideal for imaging subcellular structures deep within living tissues. Two-photon microscopy overcomes these limitations and great breakthrough has been obtained when applied to monitor microglial dynamics in vivo. ${ }^{56}$ This mini-review provides an update, mostly from an imaging perspective, on the morphological transformation, proliferation and polarisation during microgliosis in ischaemic stroke, and attempts to uncover the link between microglial activation and their potential functions.

\section{Morphological transformation of microglia after ischaemic stroke}

Microglia in a healthy brain display ramified morphology. The fine processes of microglia are highly dynamic and can constantly monitor the CNS microenvironment to serve a housekeeping function. ${ }^{51920}$ In response to pathological stimuli, microglia change their 
process dynamics and undergo a rapid morphological transformation. Microglia can make targeted movement toward the injured site by retracting or extending their processes. ${ }^{5}$ Ramified microglia transform into amoeboid microglia through a stereotypical sequence of events. ${ }^{21}$ Microglia are sensitive to blood flow fluctuation. The dynamics of their processes is highly correlated with local blood supply, and reduction in blood flow induces a significant decrease in process activity. ${ }^{15}$ In addition, microglia tend to retract their branches after ischaemia, leading to a reduction in the total length and the total number of microglial processes. ${ }^{22}$ Loss of blood flow in the peri-infarct region results in marked deramification and amoeboid transformation of soma. Interestingly, the presence of residual blood flow appears to be necessary for the motility of microglial processes since complete loss of blood flow arrested the morphological transformation of microglia. ${ }^{15}$ In addition to blood flow change, other events occurred during ischaemia also contribute to the activation of microglia. Our recent data showed that blood-brain barrier leakage can cause marked microglial deramification without a significant increase in mRNA expression level of inflammatory cytokines, indicating that morphological transformation occurs earlier than inflammatory response. ${ }^{22}$ The degree of morphological transformation of activated microglia is correlated to the severity and duration of ischaemia. Transient ischaemia only induces a moderate reduction in the total length and the total number of microglial processes, and this partial deramification and reduction in process dynamics can be reversed following reperfusion. ${ }^{1522}$ In contrast, severe ischaemia leads to a complete deramification and microglia transform into amoeboid shapes resembling peripheral macrophage. ${ }^{23}$ In addition, there is a regional difference in microglial activation during ischaemia. In focal cerebral ischaemia, microglia branching complexity is found to be increased in the striatal region but decreased in cortical regions. ${ }^{24}$ Thus, microglia can be hyper-ramified or deramified in response to focal ischaemia depending on brain regions or the degree of ischaemia.

The activated microglia display distinctive spatial and temporal patterns in region spanning the peri-infarct tissue. ${ }^{25}{ }^{26}$ In focal ischaemia, the area of microgliosis can be divided into three different zones: core zone, accumulation zone and marginal zone. ${ }^{23}$ Microglia in different zones have distinct morphological characteristics. Ramified microglia are located in the cortical region with normal blood flow, microglia with few short and stout processes are observed in the marginal zone, and amoeboid microglia with hypertrophic cell body are found in the accumulation zone and the core region. ${ }^{23}$ The distribution pattern of microglia with different morphology across the peri-infarct region suggests that morphological transformation of microglia may reflect a transition in microglial function, and distinct morphological phenotypes may represent different pathological states of ischaemic tissue.

\section{Proliferation of microglia after ischaemia}

In a healthy brain, the number of microglia remains relatively steady and only a small percentage of cells proliferate at a given time. ${ }^{42728}$ However, microglia rapidly transform into activated state and undergo active division following ischaemia. ${ }^{23} 29$ Proliferative activity of microglia after ischaemia is associated with the severity of ischaemia, and microglia in different brain regions may have different sensitivity to ischaemia. Massive expansion of microglia in hippocampal CA1 region can be observed after transient global ischaemia, but transient global ischaemia does not induce significant proliferation of microglia in cerebral cortices. $^{22}$ In focal ischaemia, the activated microglia accumulate in the peri-infarct region and adapt deramified morphology, and proliferative activity of microglia mainly occurs in the accumulation zone where microglia have the highest density (based on visibly higher numbers of bromodeoxyuridine (BrdU)-positive microglia and quantitative evaluation of microglial density in the accumulation zone) ${ }^{23}$

There are still some controversies regarding the origins of microglia involved in microgliosis after ischaemia. Previous studies suggest that there are at least three different origins for microgliosis after ischaemic stroke: local self-renewal of reactive microglia, infiltration of blood-derived cells and mobilisation of pericytes or other endogenous progenitors. ${ }^{16}$ Earlier studies suggest that haematopoietic cells transplanted into lethally irradiated mice infiltrate into the brain parenchyma and differentiate into microglia after middle cerebral artery occlusion. ${ }^{30} 31$ These results offer a hope that bone marrow-derived microglial precursors can be used as a potential candidate of cell-based therapy for ischaemic stroke. A more recent study by Ozen et al indicates that microglia can be derived from pericytes after ischaemia. ${ }^{32}$ Using a photothrombosis stroke model, our study suggests that although circulating cells can infiltrate the cerebral parenchyma after stroke, these blood-derived cells are different from reactive microglia and proliferation of resident microglia is the main source of microgliosis. ${ }^{23}$ Interestingly, Lambertsen and colleagues suggest that reactive microglia in the hippocampus have a mixed origin after ischaemia. ${ }^{33}$ This study suggests that reactive microglia in ischaemic mice can originate from infiltrating cells or resident microglia, but they only derived from resident microglia in ischaemic rat. Thus, microglia may derive from different origins depending on the experimental models or pathological conditions.

Microglia from different origins may have distinct phenotypes and functional properties. It remains to be determined how microglia from different origins affect the progression of ischaemic injury. A comprehensive understanding of the regulatory mechanisms of microgliosis will help unravel the precise role of microglia in ischaemic stroke. In addition, controlling the source of microglia may be an effective strategy for stroke treatment. 


\section{Polarisation of microglia after ischaemic stroke}

In addition to morphological transformation, the activation of microglia involves a phenotypic shift in gene expression or function. Activated microglia following ischaemia can produce a variety of effector molecules including proinflammatory cytokines, anti-inflammatory cytokines and growth factors. ${ }^{934}$ Early efforts attempt to classify the activated microglia into two major phenotypes: M1 and M2 phenotypes. However, recent studies have revealed that the polarisation states of microglia are much more diverse than the M1/M2 polarisation. ${ }^{95}$ In fact, the expressions of proinflammatory cytokines and anti-inflammatory cytokines are not mutually exclusive and microglia may not be simply classified only in two opposite phenotypes. Microglia can express distinct transcription profiles in response to pathological conditions, and a variety of intermediate phenotypes beyond M1/ M2 concepts exists. ${ }^{36}$ Microglia/macrophage display a distinctive spatial pattern of their phenotype markers at given time points after ischaemia, but the expression of these markers changes over time..$^{25}$ Microglia and/ or macrophage can switch from an early anti-inflammatory phenotype to a proinflammatory phenotype during ischaemic progression. ${ }^{38}{ }^{39}$ Thus, activated microglia are plastic and can experience a phenotypic transition during ischaemic progression. A variety of signalling molecules are involved in shaping the polarisation state of microglia. For examples, lipopolysaccharide and interferon- $\gamma$ can induce microglial differentiation toward a proinflammatory phenotype, but CXCL16 and interleukin 4 promote anti-inflammatory polarisation. ${ }^{40-42}$ Some of the molecules driving microglia polarisation have been studied in great detail and we refer the readers to several excellent recent reviews focused on molecular mechanisms underlying the phenotypic switch. ${ }^{34} 4344$

Activated microglia are involved in brain inflammation, debris clearance, astrocyte activation and synapse remodelling. ${ }^{85}$ Although a lot of effort has been made to modulate microglial polarisation toward beneficial phenotype,$^{44}{ }^{46}$ the exact role of microglial polarisation and the relative contributions of different microglial phenotypes in ischaemic stroke are still not completely understood. The diverse polarisation phenotypes imply that microglia may participate in various pathological processes, and a phenotype switch may reflect a transition in microglial function. Previous studies aiming to suppress or deplete microglia obtain different results. Some studies show that microglial activation is neurotoxic after ischaemic stroke, ${ }^{17} 47$ but others suggest that microglial activation is neuroprotective because elimination of microglia exacerbates ischaemic injury. ${ }^{18} 4849$ Microglia activation not only contributes to pathological mechanisms but also contributes to tissue repair mechanisms ${ }^{10}$ and it is conceivable that different polarisation phenotypes of microglia may contribute to different aspects of pathology. Experimental manipulation intent to suppress the neurotoxic effect of microglia could also compromise the beneficial functions. Considering the diverse functions of microglia, perhaps homeostatic maintenance of different polarisation phenotypes should be considered in future therapeutic strategies.

\section{CONCLUSION}

Microglia are highly sensitive to pathological changes in ischaemic stroke. Recent studies have revealed that the activation of microglia involves several fundamental changes including morphological transformation, proliferation and polarisation. Activated microglia may have multiple origins and microglia with different morphological and polarisation phenotypes may have distinct functions. Future studies will be needed to elucidate the roles of different phenotypes in neuroinflammation and pathological progression of ischaemic tissue.

Acknowledgements The author thanks Muhammad Tariq Hafeez for critical reading of the manuscript.

Contributors SZ is the sole author of this manuscript.

Funding This work was supported by grants from the National Natural Science Foundation of China (Nos. 31471045 and 81771324).

Competing interests None declared.

Patient consent for publication Not required.

Provenance and peer review Commissioned; externally peer reviewed. Data sharing statement No additional data are available.

Open access This is an open access article distributed in accordance with the Creative Commons Attribution Non Commercial (CC BY-NC 4.0) license, which permits others to distribute, remix, adapt, build upon this work non-commercially, and license their derivative works on different terms, provided the original work is properly cited, appropriate credit is given, any changes made indicated, and the use is non-commercial. See: http://creativecommons.org/licenses/by-nc/4.0/.

\section{REFERENCES}

1. Alliot F, Godin I, Pessac B. Microglia derive from progenitors, originating from the yolk sac, and which proliferate in the brain. Brain Res Dev Brain Res 1999;117:145-52.

2. Ginhoux F, Greter M, Leboeuf M, et al. Fate mapping analysis reveals that adult microglia derive from primitive macrophages. Science 2010;330:841-5.

3. Huang $\mathrm{Y}, \mathrm{Xu} \mathrm{Z}$, Xiong S, et al. Repopulated microglia are solely derived from the proliferation of residual microglia after acute depletion. Nat Neurosci 2018;21:530-40.

4. Askew K, Li K, Olmos-Alonso A, et al. Coupled Proliferation and Apoptosis Maintain the Rapid Turnover of Microglia in the Adult Brain. Cell Rep 2017;18:391-405.

5. Davalos D, Grutzendler J, Yang G, et al. ATP mediates rapid microglial response to local brain injury in vivo. Nat Neurosci 2005;8:752-8.

6. Nimmerjahn A, Kirchhoff F, Helmchen F. Resting microglial cells are highly dynamic surveillants of brain parenchyma in vivo. Science 2005;308:1314-8.

7. Weinhard L, di Bartolomei G, Bolasco G, et al. Microglia remodel synapses by presynaptic trogocytosis and spine head filopodia induction. Nat Commun 2018;9:1228.

8. Parkhurst CN, Yang G, Ninan I, et al. Microglia promote learningdependent synapse formation through brain-derived neurotrophic factor. Cell 2013;155:1596-609.

9. Ma Y, Wang J, Wang Y, et al. The biphasic function of microglia in ischemic stroke. Prog Neurobiol 2017;157:247-72.

10. Colonna M, Butovsky O. Microglia Function in the Central Nervous System During Health and Neurodegeneration. Annu Rev Immunol 2017;35:441-68.

11. Hernandez-Ontiveros DG, Tajiri N, Acosta S, et al. Microglia activation as a biomarker for traumatic brain injury. Front Neurol 2013;4:30.

12. Yin Z, Raj D, Saiepour $N$, et al. Immune hyperreactivity of $A \beta$ plaque-associated microglia in Alzheimer's disease. Neurobiol Aging 2017;55:115-22. 
13. Gómez-Nicola D, Fransen NL, Suzzi S, et al. Regulation of microglial proliferation during chronic neurodegeneration. $J$ Neurosci 2013;33:2481-93.

14. Lull ME, Block ML. Microglial activation and chronic neurodegeneration. Neurotherapeutics 2010;7:354-65.

15. Masuda T, Croom D, Hida H, et al. Capillary blood flow around microglial somata determines dynamics of microglial processes in ischemic conditions. Glia 2011;59:1744-53.

16. Li T, Zhang S. Microgliosis in the Injured Brain: Infiltrating Cells and Reactive Microglia Both Play a Role. Neuroscientist 2016;22:165-70.

17. Yenari MA, Xu L, Tang XN, et al. Microglia potentiate damage to blood-brain barrier constituents: improvement by minocycline in vivo and in vitro. Stroke 2006;37:1087-93.

18. Szalay G, Martinecz B, Lénárt N, et al. Microglia protect against brain injury and their selective elimination dysregulates neuronal network activity after stroke. Nat Commun 2016;7:11499.

19. Kabba JA, Xu Y, Christian H, et al. Microglia: Housekeeper of the Central Nervous System. Cell Mol Neurobiol 2018;38:53-71.

20. Nayak D, Roth TL, McGavern DB. Microglia development and function. Annu Rev Immunol 2014;32:367-402.

21. Stence N, Waite M, Dailey ME. Dynamics of microglial activation: a confocal time-lapse analysis in hippocampal slices. Glia 2001;33:256-66

22. Ju F, Ran Y, Zhu L, et al. Increased BBB Permeability Enhances Activation of Microglia and Exacerbates Loss of Dendritic Spines After Transient Global Cerebral Ischemia. Front Cell Neurosci 2018;12:236

23. Li T, Pang S, Yu Y, et al. Proliferation of parenchymal microglia is the main source of microgliosis after ischaemic stroke. Brain 2013;136(Pt 12):3578-88.

24. Morrison HW, Filosa JA. A quantitative spatiotemporal analysis of microglia morphology during ischemic stroke and reperfusion. $J$ Neuroinflammation 2013;10:4

25. Perego C, Fumagalli S, De Simoni MG. Temporal pattern of expression and colocalization of microglia/macrophage phenotype markers following brain ischemic injury in mice. J Neuroinflammation $2011 ; 8: 174$

26. Schroeter M, Jander S, Huitinga I, et al. Phagocytic response in photochemically induced infarction of rat cerebral cortex. The role of resident microglia. Stroke 1997;28:382-6.

27. Füger $\mathrm{P}$, Hefendehl $\mathrm{JK}$, Veeraraghavalu $\mathrm{K}$, et al. Microglia turnover with aging and in an Alzheimer's model via long-term in vivo singlecell imaging. Nat Neurosci 2017;20:1371-6.

28. Tay TL, Mai D, Dautzenberg J, et al. A new fate mapping system reveals context-dependent random or clonal expansion of microglia. Nat Neurosci 2017;20:793-803.

29. Moraga A, Pradillo JM, García-Culebras A, et al. Aging increases microglial proliferation, delays cell migration, and decreases cortical neurogenesis after focal cerebral ischemia. J Neuroinflammation 2015;12:87

30. Hess DC, Abe T, Hill WD, et al. Hematopoietic origin of microglial and perivascular cells in brain. Exp Neurol 2004;186:134-44.

31. Priller J, Flügel $\mathrm{A}$, Wehner $\mathrm{T}$, et al. Targeting gene-modified hematopoietic cells to the central nervous system: use of green fluorescent protein uncovers microglial engraftment. Nat Med 2001;7:1356-61.

32. Özen I, Deierborg T, Miharada K, et al. Brain pericytes acquire a microglial phenotype after stroke. Acta Neuropathol 2014;128:381-96.

33. Lambertsen KL, Deierborg T, Gregersen R, et al. Differences in origin of reactive microglia in bone marrow chimeric mouse and rat after transient global ischemia. J Neuropathol Exp Neurol 2011;70:481-94.

34. Gülke E, Gelderblom M, Magnus T. Danger signals in stroke and their role on microglia activation after ischemia. Ther Adv Neurol Disord 2018;11:175628641877425.

35. Dubbelaar ML, Kracht L, Eggen BJL, et al. The Kaleidoscope of Microglial Phenotypes. Front Immunol 2018;9:9.

36. Cherry JD, Olschowka JA, O'Banion MK. Neuroinflammation and M2 microglia: the good, the bad, and the inflamed. J Neuroinflammation 2014;11:98

37. Ransohoff RM. A polarizing question: do $\mathrm{M} 1$ and $\mathrm{M} 2$ microglia exist? Nat Neurosci 2016;19:987-91.

38. Hu X, Li P, Guo Y, et al. Microglia/macrophage polarization dynamics reveal novel mechanism of injury expansion after focal cerebral ischemia. Stroke 2012;43:3063-70.

39. Rajan WD, Wojtas B, Gielniewski B, et al. Dissecting functional phenotypes of microglia and macrophages in the rat brain after transient cerebral ischemia. Glia 2019;67.

40. Lepore F, D'Alessandro G, Antonangeli F, et al. CXCL16/CXCR6 Axis Drives Microglia/Macrophages Phenotype in Physiological Conditions and Plays a Crucial Role in Glioma. Front Immunol 2018:9:2750.

41. Vay SU, Flitsch LJ, Rabenstein M, et al. The plasticity of primary microglia and their multifaceted effects on endogenous neural stem cells in vitro and in vivo. J Neuroinflammation 2018;15:226.

42. Liu X, Liu J, Zhao S, et al. Interleukin-4 Is Essential for Microglia/ Macrophage M2 Polarization and Long-Term Recovery After Cerebral Ischemia. Stroke 2016;47:498-504.

43. Fumagalli M, Lombardi $\mathrm{M}$, Gressens $\mathrm{P}$, et al. How to reprogram microglia toward beneficial functions. Glia 2018;66:2531-49.

44. Wang J, Xing H, Wan L, et al. Treatment targets for M2 microglia polarization in ischemic stroke. Biomed Pharmacother 2018; $105: 518-25$.

45. Gomez-Nicola D, Perry VH. Microglial dynamics and role in the healthy and diseased brain: a paradigm of functional plasticity. Neuroscientist 2015;21:169-84.

46. Xia CY, Zhang S, Gao Y, et al. Selective modulation of microglia polarization to M2 phenotype for stroke treatment. Int Immunopharmacol 2015;25:377-82.

47. Franco EC, Cardoso MM, Gouvêia A, et al. Modulation of microglial activation enhances neuroprotection and functional recovery derived from bone marrow mononuclear cell transplantation after cortical ischemia. Neurosci Res 2012;73:122-32.

48. Jin WN, Shi SX, Li Z, et al. Depletion of microglia exacerbates postischemic inflammation and brain injury. J Cereb Blood Flow Metab 2017;37:2224-36

49. Otxoa-de-Amezaga A, Miró-Mur F, Pedragosa J, et al. Microglial cell loss after ischemic stroke favors brain neutrophil accumulation. Acta Neuropathol 2019;137. 